\title{
NUCLEATION OF QUARK MATTER IN NEUTRON STAR CORES
}

\author{
T. Harko, K. S. Cheng, and P. S. TANG \\ Department of Physics, University of Hong Kong, Pokfulam Road, Hong Kong, China; \\ harko@hkucc.hku.hk, hrspksc@hkucc.hku.hk, anisia@bohr.physics.hku.hk \\ Received 2004 January 14; accepted 2004 March 8
}

\begin{abstract}
We consider the general conditions of quark droplet formation in high-density neutron matter. The growth of the quark bubble (assumed to contain a sufficiently large number of particles) can be described by means of a Fokker-Planck equation. The dynamics of the nucleation essentially depends on the physical properties of the medium in which it takes place. The conditions for quark bubble formation are analyzed within the framework of both dissipative and nondissipative (with zero bulk and shear viscosity coefficients) approaches. The conversion time of the neutron star to a quark star is obtained as a function of the equation of state of the neutron matter and of the microscopic parameters of the quark nuclei. As an application of the formalism obtained, we analyze the first-order phase transition from neutron matter to quark matter in rapidly rotating neutron star cores, triggered by the gravitational energy released during the spinning down of the neutron star. The endothermic conversion process, via gravitational energy absorption, could take place in a very short time interval, of the order of a few tens of seconds, in a class of dense compact objects with very high magnetic fields, called magnetars.
\end{abstract}

Subject headings: dense matter — pulsars: general — stars: interiors — stars: neutron

\section{INTRODUCTION}

Since Witten (1984), following early proposals by Itoh (1970) and Bodmer (1971), suggested that strange quark matter, consisting of $u$-, $d$-, and $s$-quarks, is energetically the most favorable state of matter, the problem of the existence of strange quark stars has been intensively investigated in the physical and astrophysical literature. The possibility that some compact objects could be strange stars remains an interesting and intriguing, but still open, question. Witten (1984) also proposed two possible formation scenarios for strange matter: the quark-hadron phase transition in the early universe, and conversion of neutron stars into strange ones at ultrahigh densities. In the theories of strong interaction, quark bag models suppose that breaking of the physical vacuum takes place inside hadrons. As a result, vacuum energy densities inside and outside a hadron become essentially different, and the vacuum pressure on the bag wall equilibrates the pressure of quarks, thus stabilizing the system. If the hypothesis of quark matter is true, then some neutron stars could actually be strange stars, made entirely of strange matter (Alcock et al. 1986; Haensel et al. 1986). However, there are general arguments against the existence of strange stars (Caldwell \& Friedman 1991). For an extensive review of strange star properties, see Cheng et al. (1998a).

Several mechanisms have been proposed for the formation of quark stars. Quark stars are expected to form during the collapse of the core of a massive star after a supernova explosion, as a result of a first- or second-order phase transition, resulting in deconfined quark matter (Dai et al. 1995). The proto-neutron star core, or the neutron star core, is a favorable environment for the conversion of ordinary matter to strange quark matter (Cheng et al. 1998b). Another possibility is that some neutron stars in low-mass X-ray binaries can accrete sufficient mass to undergo a phase transition to become strange stars (Cheng \& Dai 1996). This mechanism has also been proposed as a source of radiation emission for cosmological gamma-ray bursts (Cheng \& Dai 1998). Some basic properties of strange stars, such as mass, radius, cooling, collapse, and surface radiation, have also been studied (Cheng \& Harko 2000, 2003; Harko \& Cheng 2000, 2002; Ng et al. 2003).

The physical mechanisms of the transition from neutron matter to quark matter in an astrophysical background have been studied in several models. The first is that of Olinto (1987), who used a nonrelativistic diffusion model. As such, this is a slow combustion model, with the burning front propagating at a speed of approximately $10 \mathrm{~m} \mathrm{~s}^{-1}$. This is determined primarily by the rate at which one of the down quarks inside the neutrons is converted, through a weak decay, to a strange quark: $d+u \rightarrow s+u$. The second method of describing the conversion process was first suggested by Horvath \& Benvenuto (1988) and analyzed in detail by Lugones et al. (1994) and Lugones \& Benvenuto (1995), who modeled the conversion as a detonation. In this case the conversion rate is several orders of magnitude faster than that predicted by the slow combustion model. This model is based on the relativistic shock waves and combustion theory. But regardless of the way in which the transformation occurs, an initial seed of quark matter is needed to start the process.

Real neutron stars have two conserved charges: electric and baryonic. Therefore, a neutron star has more than one independent component, and in this sense is a "complex" system (Glendenning 1992). The characteristics of a first-order phase transition, such as the deconfinement transition, are very different in the two cases. In the complex system the conserved charges can be shared by the two phases in equilibrium in different concentrations in each phase. The mixed phase, formed from hadrons and quarks, cannot exist in a simple body in the presence of gravity because the pressure in that phase is a constant (Glendenning 2000). This causes a discontinuity in the density distribution in the star occurring at the radius where Gibbs criteria are satisfied. The isospin symmetry energy in neutron-rich matter will exploit the degree of freedom of readjusting the charges between hadronic and quark phases in equilibrium so as to reduce the symmetry energy to an extent consistent with charge conservation. Regions of hadronic matter will have a net positive charge 
neutralized by a net negative charge on the quark matter regions (Glendenning 2000). Coulomb repulsion will prevent the regions of like charge from growing too large, and the surface energy will act in the opposite sense in preventing them from becoming too small. Consequently, the mixed phase will form a Coulomb lattice so as to minimize the sum of Coulomb and surface interface energy at each proportion of phases. As the quark phase becomes more abundant, the droplets merge to form rods, rods merge to form slabs, etc. (Glendenning 2000). Hence, the actual geometric phase of quark nuggets in a neutron star evolves as a function of the pressure and of the surface energy between dense nuclear matter and quark matter.

In order to describe the process of the formation and evolution of quark nuclei in neutron stars, one must use nucleation theory. The goal of nucleation theory is to compute the probability that a bubble or droplet of the $A$ phase appears in a system initially in the $B$ phase near the critical temperature (Landau \& Lifshitz 1980). Homogeneous nucleation theory applies when the system is pure. Nucleation theory is applicable for first-order phase transitions when the matter is not dramatically supercooled or superheated. If substantial supercooling or superheating is present, or if the phase transition is second order, then the relevant dynamics is spinodal decomposition (Shukla \& Mohanty 2001).

The nucleation of strange quark matter inside hot, dense nuclear matter was investigated, using Zel'dovich's kinetic theory of nucleation, by Horvath et al. (1992). By assuming that the newly formed strange quark matter bubble can be described by a simple bag model containing $N_{q}$ ultrarelativistic quarks, and by assuming that the time evolution of the radius of the bubble is described by an equation of the form $d r / d t=$ $\left(r-R_{c}\right) / \tau_{w}$, where $R_{c}$ is the critical radius of the bubble and $\tau_{w}$ is the weak interaction timescale, the nucleation rate $\xi$ is given by

$$
\xi=2.2 \times 10^{-2}(T / \sigma)^{1 / 2} N_{\mathrm{cq}}^{3 / 4} \tau_{w}^{-1} \exp \left(-3.1 N_{\mathrm{cq}}^{1 / 2} \sigma / T\right),
$$

where $N_{\mathrm{cq}} \leq 300$ is the critical quark number, $\sigma$ is the surface tension of the bubble, and $n_{n}$ and $n_{q}$ are the particle number densities in the neutron and quark phase, respectively (Horvath et al. 1992). In this way a lower bound for the temperature of the nucleation, $T \geq 2.1 \mathrm{MeV}$, can be obtained. The effects of the curvature energy term on thermal strange quark matter nucleation have been considered by Horvath (1994), who derived, within the same approach, the following expression for the nucleation rate:

$$
\xi=\frac{r_{c}^{2} n_{q} n_{n}}{4 \pi \tau_{w}}\left(\frac{T}{\sigma}\right)^{1 / 2} \exp \left(\frac{-4 \pi R_{c}^{2}}{3 T}\right) .
$$

All the effects of the curvature enter only through the value of $R_{c}$. As a general conclusion, we find that even though the curvature term acts against strange quark matter nucleation, the physical temperature of a just-born proto-neutron star is, in any model, more than enough to drive an efficient boiling of the neutron material (the observations of the neutrino flux from SN 1987A are consistent with an effective temperature of $T \sim 4 \mathrm{MeV}$ ). The possibility of stable strange quark matter (both bulk and quasi-bulk) at finite temperature, and some of its properties, have been investigated within the framework of the dynamical density-dependent quark mass model of confinement by Chakrabarty (1993). The behavior of the surface tension and the stability of quark droplets at $T \neq 0$ have also been discussed, with reference to strangelet formation in ultrarelativistic heavy-ion collisions.

A different approach to the problem of thermal nucleation was taken by Olesen \& Madsen (1994). They used the assumption (standard in the theory of bubble nucleation in firstorder phase transitions) that bubbles form at a rate given by $R \approx T^{4} \exp \left(-W_{c} / T\right)$, where $W_{c}$ is the minimum work required to form a bubble with radius $r_{c}$. As a main result it follows that if the bag constant lies in the interval where threeflavor but not two-flavor quark matter is stable at zero pressure and temperature $\left(145 \mathrm{MeV} \leq B^{1 / 4} \leq 163 \mathrm{MeV}\right)$, then all or parts of a neutron star will be converted into strange matter, during the first second of its existence. For the bag constant above the stability interval, a partial transformation is still possible (Olesen \& Madsen 1994).

Heiselberg (1995) calculated the rate of formation of quark matter droplets in neutron stars from a combination of bubble formation rates in cold degenerate and high-temperature matter, taking into account nuclear matter calculations of the viscosity and thermal conductivity. The droplet formation rate is that of Langer \& Turski (1973), given by $I=(k / 2 \pi)$ $\Omega_{0} \exp \left(-W_{c} / T\right)$, where $\Omega_{0}=(2 / 3 \sqrt{3})(\sigma / T)^{3 / 2}\left(r_{c} / \xi_{q}\right)^{4}$ is the statistical prefactor, which measures the phase-space volume of the saddle point around $R_{c}$ that the droplet has to pass on its way to the lower energy state. Here $\xi_{q}$ is the quark correlation length. The dynamical prefactor determines the droplet growth rate and is given by $k=\left(2 \sigma / \Delta w^{2} r_{c}^{3}\right)[\lambda T+$ $2(4 \eta / 3+\zeta)]$, where $\Delta w$ is the enthalpy difference and $\lambda, \eta$, and $\zeta$ are the thermal conductivity and the shear and bulk viscosities, respectively. The droplet formation rate can be expressed as (Heiselberg 1995)

$I=\frac{\sigma_{20}^{7 / 2} \mu_{400}^{2} \eta_{50}}{\Delta P_{10} \Delta w_{10}^{2} T_{10}^{3 / 2}} \exp \left(185-134 \frac{\sigma_{20}^{3}}{\Delta P_{10}^{2} T_{10}}\right) \mathrm{s}^{-1} \mathrm{~km}^{-3}$,

where $\mu_{400}$ is the quark chemical potential in units of $400 \mathrm{MeV}$.

The total number $N$ of droplets formed by nucleation is the integrated rate over the volume of the neutron star and the time after the nucleation process starts at the moment $t_{0}$, $N=\int_{0}^{R} 4 \pi r^{2} d r \int_{t_{0}}^{\infty} I(\Delta p(r), T(t)) d t$, where $R$ is the radius of the neutron star. The pressure and temperature depend sensitively on the equation of state (EOS) of the nuclear and quark matter. To convert the core of the neutron star into quark matter, at least one droplet must be formed, i.e., $N>1$. Then, by equation (3), the condition for the formation of at least one droplet requires $\sigma \leq 24 \mathrm{MeV} \mathrm{fm}^{-2}\left(\Delta P_{c, 10}^{2 / 3} T_{c, 10}^{1 / 3}\right)$, where $P_{c}$ and $T_{c}$ are the core values of the pressure and temperature, respectively (Heiselberg 1995). The pressure and enthalpy difference depends strongly on the EOS of nuclear and quark matter, and hence the droplet formation rate cannot be reliably estimated.

The effect of subcritical hadron bubbles on an inhomogeneous first-order quark-hadron phase transition was studied in Shukla et al. (2000). The transition from nuclear matter (consisting of neutrons, protons, and electrons) to matter containing strangeness was considered, within a mean field type model and using Langer nucleation theory, by Norsen (2002). An estimate of the time it takes for the new phase to appear at various densities and times in the cooling history of a proto-neutron star was also given.

On the other hand, as a result of an increase of the central density of the star, due, for example, to accretion or spinning down, a metastable, supercompressed neutron phase can 
appear, with a star consisting of the new phase surrounded by normal neutron matter (Grassi 1998). This situation is similar to a gas undergoing a phase transition to its liquid state if compressed to a volume $V_{c}$ at fixed $T$. If it is slowly compressed, it may stay in the vapor state even for $V<V_{c}$ (similar examples are liquid water freed of dissolved air, which can be heated above boiling temperature without the formation of vapor and cooled below freezing temperature without solidification). The transition from the supercompressed neutron phase to the quark phase can take place over a very long time, which in some cases can be longer than the estimated life of the neutron star (Grassi 1998). Therefore, an increase in the density of the neutron star does not automatically lead to the transition to quark matter inside the star.

The effect of the magnetic field on the quark star structure and on the nucleation process of the quark bubbles has been studied in Chakrabarty $(1995,1996)$ and Chakrabarty \& Sahu (1996). In the presence of strong magnetic fields the EOS of strange quark matter changes significantly. The strange stars become more compact, the magnetic field reducing the mass and radius of the star. The surface energy and the curvature term of the quark phase both diverge, with the surface tension diverging logarithmically, while the curvature term diverges much faster. Therefore, the thermal nucleation of quark bubbles in a compact metastable state of neutron matter is completely forbidden in the presence of a strong magnetic field.

These results for the formation of quark bubbles in neutron matter have been obtained by using the Csernai \& Kapusta theory of nucleation (Csernai \& Kapusta 1992a, 1992b) and its extension including the thermal conductivity of dense matter (Venugopalan \& Vischer 1994). In this theory both the hadron and quark materials are considered as substances with zero baryonic number and are treated by using a relativistic formalism. The principal result of this approach is the suggestion that the prefactor $k$ is proportional to the transport coefficients (viscosity and thermal conductivity) of the neutron matter; thus, when these coefficients vanish, a quark bubble necessarily does not form.

However, Ruggeri \& Friedman (1996) argued that the energy flow does not vanish in the absence of any heat conduction or viscous damping. Since the change of energy density $e$ in time is given, in the low-velocity limit, by the conservation equation $\partial e / \partial t=-\nabla(w \boldsymbol{v})$ (Csernai \& Kapusta 1992a), where $w$ is the enthalpy and $\boldsymbol{v}$ is the velocity, this implies that the energy flow $\sim w \boldsymbol{v}$ is always present. Therefore, an expression for the prefactor can be derived that does not vanish in the absence of viscosity. The viscous effects cause only a small perturbation to the prefactor. The differences between the Csernai-Kapusta (CK; Csernai \& Kapusta 1992a) and Ruggieri-Friedman (RF; Ruggeri \& Friedman 1996) results are due to the technical differences in the treatment of the pressure gradients.

A generalized approach, following the CK formalism and leading to the prefactor in both viscous and nonviscous regimes, was developed by Shukla et al. (2001). Unlike in Csernai \& Kapusta (1992a), the linearized relativistic hydrodynamics equations have been solved in all regions.

It is the purpose of the present paper to extend the Zel'dovich nucleation theory for the formation of quark droplets in neutron matter, by taking into account the effects of both the energy flow and thermal conductivity and shear and bulk viscosities. As a result, a more general expression for the quark matter droplet rate formation can be obtained, which is also valid in the limiting case of vanishing conductivity and viscosity coefficients. In the nucleation theory of Csernai \& Kapusta (1992a), the transition from the neutron to quark state is possible only for normal matter having viscous properties. For zero bulk and shear viscosities the transition is impossible. This assumption is, however, too restrictive, and the transition can also take place for a perfect (no viscosity or heat conduction) neutron matter fluid. We also derive the expression of the rate formation for this case.

An alternative point of view of the transformation of neutron matter into strange matter can be developed if one assumes that the conversion process is endothermic. In this case the strange quark matter formation inside a neutron star is triggered by an external source of energy (accretion from the companion star or spin-down). Pulsars are born with an enormous angular momentum and rotational energy, which they radiate over a long period of time via electromagnetic radiation and electron-positron pair emission. When rotating rapidly, a pulsar is centrifugally flattened. With decreasing angular velocity the central density of the star is increasing and may attain the critical density necessary for a phase transition. First at the center and then in an expanding region, the neutron matter will be converted to a highly compressible quark matter phase (Glendenning et al. 1997). The conversion of neutron matter to quark matter alters the moment of inertia of the star, and the epoch over which conversion takes place will be signaled in the spin-down characteristics of the pulsar. A measurable quantity, the braking index $\Omega \ddot{\Omega} / \dot{\Omega}^{2}$, where $\Omega$ is the angular velocity of the star, could be an observational indicator of the slow transition from neutron to quark phase (Glendenning et al. 1997). By using the formalism developed for the nucleation of quark matter, we reconsider the idea of phase transition from neutron matter to quark matter in rotating compact stellar objects, by assuming that the nucleation and the growth of the quark droplets and the formation of a quark core in a neutron star are a result of the transfer of the gravitational energy to the quark droplets, as a result of the spinning down of the rapidly rotating neutron star.

The present paper is organized as follows. The nucleation kinetics of strange matter bubbles is considered, within the framework of the Zel'dovich nucleation theory, in $\S 2$. The expressions for the rate of formation of quark matter are derived, in both the perfect and dissipative neutron fluid case, in $\S 3$. The transition of a neutron star to a quark star, due to increase of the central density as a result of the spinning down, is considered in $\S 4$. In $\S 5$ we discuss our results and give conclusions.

\section{NUCLEATION KINETICS OF STRANGE MATTER IN NEUTRON STARS}

We consider that the change from the metastable neutron phase to the stable quark phase occurs as the result of fluctuations in a homogeneous medium, formed of neutrons, in which small quantities of the quark phase (called bubbles or nuclei) are randomly generated. Since the process of creation of an interface is energetically unfavorable, it follows that when a quark nucleus is below a certain size, it is unstable and disappears again. Surface effects disfavor the survival of small bubbles below the radius $R_{c}$ (called critical size; the nuclei of this size are called critical nuclei or bubbles), which is nothing but the value that extremizes the thermodynamical work $W$ necessary to create the bubbles. Only nuclei whose size $r$ is above the value $R_{c}$ are stable and can survive (Landau \& Lifshitz 1980). The nuclei are assumed to be macroscopic objects containing a large number of particles (quarks). 
For strongly degenerate neutron matter, the thermodynamic work $W$ necessary to create a quark bubble is (Alcock \& Olinto 1989; Mardor \& Svetitsky 1991; Madsen \& Olesen 1991; Olesen \& Madsen 1994; Horvath 1994)

$W=\left[n_{q}\left(\mu_{q}-\mu_{n}\right)-\left(P_{q}-P_{n}\right)\right] \frac{4 \pi}{3} r^{3}+4 \pi \sigma r^{2}+8 \pi \gamma r+E_{c}$,

where $P_{q}$ and $P_{n}$ are the pressures of the quark matter and neutron matter, respectively (exterior to the bubble, assumed to be spherical), $\sigma$ is the surface tension, $n_{q}$ is the particle number density of quark matter, $\mu_{q}$ and $\mu_{n}$ are the chemical potentials of each phase, $\gamma$ is the curvature coefficient, and $E_{c}=$ $\frac{3}{5} Z^{2} e^{2} / r$ is the Coulomb energy of the droplet (Heiselberg et al. 1993). Here $Z e=\left(\rho_{q}-\rho_{n}\right) V_{d}$ is the excess charge of the droplet with volume $V_{d}$ compared with the surrounding medium, and $\rho_{q}$ and $\rho_{n}$ are the charge densities of the quark and nuclear matter, respectively. Therefore, the Coulomb energy of the droplet is given by $E_{c}=16 \pi^{2}\left(\rho_{q}-\rho_{n}\right)^{2} r^{5} / 15$. Assuming that the quark matter is immersed in a uniform background of electrons and that it is in $\beta$ equilibrium, with $\mu_{d}=\mu_{s}=\mu_{u}+\mu_{e}$, it follows that the total electric charge in the quark phase can be expressed as $\rho_{q} \approx e \mu_{q}\left(m_{s}^{2} / 2-\right.$ $\left.2 \mu_{e} \mu_{q}\right) / \pi^{2}$, where $\mu_{u} \approx \mu_{d} \equiv \mu_{q}$ (Heiselberg et al. 1993). Generally, $\rho_{q} \gg \rho_{n}$.

The appropriate form of the curvature energy can be obtained from (Madsen 1993; Heiselberg et al. 1993)

$$
\begin{aligned}
E_{\mathrm{curv}} & =\frac{g r}{3 \pi} \int_{0}^{\infty} d k k\left[1+\exp \left(k-\frac{\mu}{T}\right)\right]^{-1} \\
& =\frac{g \mu^{2} r}{6 \pi}\left[1+O\left(\frac{T}{\mu}\right)^{2}\right]
\end{aligned}
$$

where $g$ is the statistical weight and $\mu$ is the chemical potential. Hence, the curvature coefficient is given by $\gamma=3 \mu^{2} / 8 \pi^{2} \sim$ $18 \mathrm{MeV} \mathrm{fm}^{-1}(\mu / 300 \mathrm{MeV})^{2}$ (Heiselberg et al. 1993).

Requiring $W$ to be an extreme, $\partial W / \partial r=0$, gives the following equation for the value of the critical radius $r=R_{c}$ :

$$
\begin{aligned}
4 \pi & {\left[n_{q}\left(\mu_{q}-\mu_{n}\right)-\left(P_{q}-P_{n}\right)\right] r^{2}+8 \pi \sigma r+8 \pi \gamma } \\
& =-\frac{16 \pi^{2}}{3}\left(\rho_{q}-\rho_{n}\right)^{2} r^{4} .
\end{aligned}
$$

By neglecting the Coulomb energy, we obtain for the critical radius $R_{c 0}$ of the bubble the expression

$$
R_{c 0}=\frac{\sigma}{C}\left(1+\sqrt{1+b_{0}}\right)
$$

where

$C=-n_{q}\left(\mu_{q}-\mu_{n}\right)+\left(P_{q}-P_{n}\right)=-n_{q} \Delta \mu+\Delta P>0$

and

$$
b_{0}=2 C|\gamma| / \sigma^{2}>0
$$

The parameter $b_{0}$ can also be represented as $b_{0} \approx$ $6\left(C / 20 \mathrm{MeV} \mathrm{fm}^{-3}\right)\left(\gamma / 18 \mathrm{MeV} \mathrm{fm}^{-1}\right)\left[\sigma /(7 \mathrm{MeV})^{3}\right]^{-2}$ (Horvath 1994).

To find the value of the critical radius for a nonnegligible electrostatic energy of the bubble, we use an iterative method, by substituting for $r$ in the right-hand side of equation (6) the zeroth-order approximation given by equation (7). Then, in first order, the critical radius is given by

$$
R_{c}=\frac{\sigma}{C}(1+\sqrt{1+b}),
$$

where

$$
b=\frac{C}{\sigma^{2}}\left[2 \gamma+\frac{4 \pi}{3}\left(\rho_{q}-\rho_{n}\right)^{2} R_{c 0}^{4}\right] .
$$

For values of $C=20 \mathrm{MeV} \mathrm{fm}^{-3}, \gamma=18 \mathrm{MeV} \mathrm{fm}^{-1}, \sigma$ in the range $(75-100 \mathrm{MeV})^{3}, \rho_{q} \approx-0.4 e \mathrm{fm}^{-3}$, and negligible $\rho_{n}$ the critical radius is of the order of $R_{c}=2-7 \mathrm{fm}$. The Coulomb correction has the effect of increasing the critical radius. The value $r=R_{c}$ corresponds to the limit beyond which large quantities of the quark phase begin to be formed. In fact, it is more appropriate to refer not to a limit point $r=R_{c}$, but to a critical range of values of $r$ near that point, with width $\delta r \sim(T / 4 \pi \sigma)^{1 / 2}$ (Landau \& Lifshitz 1980). The fluctuational development of nuclei in this range can still, with high probability, throw them back into the subcritical range, but nuclei beyond the critical range will inevitably develop into the new quark phase.

The minimum critical work required to form a stable quark bubble is therefore given by

$$
W_{c}=\frac{4 \pi \sigma^{3}}{3 C^{2}} F(b)\left[1+4 \pi\left(\rho_{q}-\rho_{n}\right)^{2} \frac{\sigma^{2}}{C^{3}} G\left(b, b_{0}\right)\right],
$$

where we denote

$$
F(b)=2+2(1+b)^{3 / 2}+3 b
$$

and

$$
G\left(b, b_{0}\right)=\frac{(1+\sqrt{1+b})^{5}}{2+2(1+b)^{3 / 2}+3 b}\left[\frac{1}{5}-\frac{\left(1+\sqrt{1+b_{0}}\right)^{4}}{(1+\sqrt{1+b})^{4}}\right] .
$$

Within a purely thermodynamic approach, one can pose only the problem of calculating the probability of occurrence in a medium of fluctuating nuclei of various sizes, the medium being regarded as in equilibrium. Instead of the thermodynamic probability of nucleation, it is more convenient to use the equilibrium distribution function for nuclei of various sizes existing in the medium, denoted $f_{0}(r)$. Here $f_{0} d r$ is the number of nuclei per unit volume of the medium with sizes in the range $d r$. According to the thermodynamic theory of fluctuations (Landau \& Lifshitz 1980),

$$
f_{0}(r) \sim \exp [-W(r) / T]
$$

Near $r=R_{c}$ the thermodynamic work can be expressed as

$$
\begin{aligned}
W(r)= & \frac{4 \pi \sigma^{3}}{3 C^{2}} F(b)\left[1+\frac{24 \pi}{5}\left(\rho_{q}-\rho_{n}\right)^{2} \frac{\sigma^{2}}{C^{3}} G(b)\right] \\
& -\left\{\left[12 \pi \sigma C^{3} \sqrt{1+b}-32 \pi^{2}\left(\rho_{q}-\rho_{n}\right)^{2}\right.\right. \\
& \left.\left.\times \sigma^{3}(1+\sqrt{1+b})^{3}\right]\left(3 C^{3}\right)^{-1}\right\}\left(r-R_{c}\right)^{2} .
\end{aligned}
$$


Therefore, for the equilibrium distribution function we find the expression

$$
\begin{aligned}
f_{0}(r)= & f_{0}\left(R_{c}\right) \exp \left(\left\{\left[12 \pi \sigma C^{3} \sqrt{1+b}-32 \pi^{2}\left(\rho_{q}-\rho_{n}\right)^{2}\right.\right.\right. \\
& \left.\left.\left.\times \sigma^{3}(1+\sqrt{1+b})^{3}\right]\left(3 C^{3} T\right)^{-1}\right\}\left(r-R_{c}\right)^{2}\right),
\end{aligned}
$$

where $f_{0}\left(R_{c}\right)=C_{0} \exp \left(-W_{c} / T\right), C_{0}=$ const.

In order to estimate the coefficient $C_{0}$ of the exponential in $f_{0}\left(R_{c}\right)$, we follow Landau \& Lifshitz (1980) and assume $C_{0}=n_{q} n_{n} R_{c}^{2}$, where $n_{n}$ and $n_{q}$ are the particle number densities in the neutron and quark phases, respectively. Thus, we obtain

$$
\begin{aligned}
f_{0}\left(R_{c}\right)= & n_{q} n_{n} R_{c}^{2} \exp \left\{-\frac{4 \pi \sigma^{3}}{3 C^{2} T} F(b)\right. \\
& \left.\times\left[1+4 \pi\left(\rho_{q}-\rho_{n}\right)^{2} \frac{\sigma^{2}}{C^{3}} G\left(b, b_{0}\right)\right]\right\} .
\end{aligned}
$$

Let $f(t, r)$ be the kinetic size distribution function of the nuclei. The elementary process that changes the size of a nucleus is the attachment to it, or the loss by it, of a quark droplet, and this is to be regarded as a small change, since the nuclei are considered to be macroscopic objects. Therefore, the growth of the nuclei is described by a Fokker-Planck equation (Landau \& Lifshitz 1980)

$$
\frac{\partial f(t, r)}{\partial t}=-\frac{\partial j}{\partial r}
$$

where $j=-B(\partial f / \partial r)+A f$ is the flux in the size space. Here $B$ is the nuclear size diffusion coefficient and $A$ is connected with $B$ by a relationship that follows from the fact that for an equilibrium distribution $j=0$. Therefore, we find $A=$ $-B W^{\prime}(r) / T$.

In the case of a continuous stationary phase transition process, we have $j=$ const. The constant flux is just the number of nuclei passing through the critical range per unit time per unit volume of the medium; i.e., it defines the rate of the process. With the condition of constant flux we obtain $-B f_{0}(\partial / \partial r)\left(f / f_{0}\right)=j$, giving

$$
\frac{f}{f_{0}}=-j \int \frac{d r}{B f_{0}}+\text { const. }
$$

The constant in this equation and $j$ are found from the boundary conditions for small and large $r$. The fluctuation probability increases rapidly with decreasing size, and small nuclei have a high probability of occurrence. This is expressed by the boundary condition $f / f_{0} \rightarrow 1$ as $r \rightarrow 0$. The boundary condition for large $r$ can be established by noting that above the critical range the function $f_{0}$ increases without limit, whereas the true distribution function $f(r)$ remains finite. This situation is expressed by imposing the boundary condition $f / f_{0}=0$ for $r \rightarrow \infty$. The solution that satisfies the above conditions is (Landau \& Lifshitz 1980)

$$
\frac{f}{f_{0}}=j \int_{r}^{\infty} \frac{d r}{B f_{0}}, \quad \frac{1}{j}=\int_{0}^{\infty} \frac{d r}{B f_{0}} .
$$

In these equations the integrand has a sharp maximum at $r=R_{c}$. By extending the integration with respect to $r$ from $-\infty$ to $+\infty$, one obtains for the number of viable quark nuclei formed in stationary conditions per unit time and per unit volume the expression

$$
\begin{aligned}
j= & \sqrt{\frac{12 \sigma C^{3} \sqrt{1+b}-32 \pi\left(\rho_{q}-\rho_{n}\right)^{2} \sigma^{3}(1+\sqrt{1+b})^{3}}{3 C^{3} T}} \\
& \times B\left(R_{c}\right) f_{0}\left(R_{c}\right) .
\end{aligned}
$$

Above the critical range, the distribution function is constant: having reached that point, the nucleus becomes steadily larger, with practically no change in the reverse direction. Accordingly, we can neglect the term containing the derivative $\partial f / \partial r$ in the flux, leading to $j=A f$. From the significance of the flux it follows that the coefficient $A$ acts as a velocity in size space, $A=(d r / d t)_{\text {macro }}$ (Landau \& Lifshitz 1980). Therefore, we find

$$
\begin{aligned}
B= & -\frac{T}{W^{\prime}(r)}\left(\frac{d r}{d t}\right)_{\text {macro }} \\
= & \left(( 3 C ^ { 3 } T ) \left\{2 \left[12 \pi \sigma C^{3} \sqrt{1+b}-32 \pi^{2}\left(\rho_{q}-\rho_{n}\right)^{2}\right.\right.\right. \\
& \left.\left.\left.\times \sigma^{3}(1+\sqrt{1+b})^{3}\right]\left(r-R_{c}\right)\right\}^{-1}\right)\left(\frac{d r}{d t}\right)_{\text {macro }} .
\end{aligned}
$$

The rate of growth of the bubble radius near the critical radius is given by $(d r / d t)_{\text {macro }}=k\left(r-R_{c}\right)$, where $k$ is the dynamical prefactor (Langer \& Turski 1973; Csernai \& Kapusta 1992a).

The prefactor $k$ has been evaluated, by solving the equations of relativistic fluid dynamics in all regions, in Shukla et al. (2001). The result (also taking into account heat conduction) is

$k=\sqrt{\frac{2 \sigma}{R_{c}^{3}} \frac{w_{n}}{(\Delta w)^{2}}}+\frac{1}{c_{s}^{2}} \frac{\sigma}{R_{c}^{3}(\Delta w)^{2}}\left[\lambda_{n} T+2\left(\frac{4}{3} \eta_{n}+\xi_{n}\right)\right]$,

where $c_{s}$ is a constant (the velocity of the sound in the medium around the saddle configuration) and $\lambda_{n}, \eta_{n}$, and $\xi_{n}$ are the thermal conductivity and shear and bulk viscosity coefficients of the neutron matter, respectively. The first term in the above equation is the same as obtained by Ruggeri \& Friedman (1996), corresponding to the case of nonviscous matter. The second term is similar to the results obtained by Csernai \& Kapusta (1992a) and Venugopalan \& Vischer (1994), but with a minor difference; i.e., instead of 4 there is a factor $c_{s}^{2}$ in the numerator.

\section{NEUTRON MATTER TO QUARK MATTER TRANSITION RATES}

From equation (24) for the prefactor it follows that the rate of formation of quark bubbles in neutron matter is given by

$$
\begin{aligned}
j= & \frac{n_{q} n_{n} R_{c}^{2}}{2 \pi} \sqrt{\frac{3 C^{3} T}{12 \sigma C^{3} \sqrt{1+b}-32 \pi\left(\rho_{q}-\rho_{n}\right)^{2} \sigma^{3}(1+\sqrt{1+b})^{3}}} \\
& \times\left(\sqrt{\frac{2 \sigma}{R_{c}^{3}} \frac{w_{n}}{(\Delta w)^{2}}}+\frac{\sigma\left\{\lambda_{n} T+2\left[(4 / 3) \eta_{n}+\xi_{n}\right]\right\}}{c_{s}^{2} R_{c}^{3}(\Delta w)^{2}}\right) \\
& \times \exp \left(-\left\{\left(\frac{4 \pi \sigma^{3}}{3 C^{2}}\right) f(b)\right.\right. \\
& \left.\left.\times\left[1+4 \pi\left(\rho_{q}-\rho_{n}\right)^{2}\left(\frac{\sigma^{2}}{C^{3}}\right) G\left(b, b_{0}\right)\right]\right\} T^{-1}\right) .
\end{aligned}
$$


To obtain the number $\xi$ of the net strange quark matter bubbles, the rate $j$ must be multiplied by the time interval available for prompt nucleation, $\Delta t$, and by the volume $V_{0}$, at which the nucleation can take place in the dense core (Horvath et al. 1992). We impose the condition that at least one quark bubble appears (which would suffice to convert the whole neutron star). Therefore, using equation (25), one obtains the following general condition for a quark bubble formation in a neutron star core:

$$
\xi=j \Delta t \Delta V_{0} \geq 1 \text {. }
$$

The quark bubble formation rate essentially depends on the enthalpy difference in the two phases. In the density range of interest, all the neutron matter EOS can be very well parameterized as polytropes, $P_{n}=K n_{B}^{\gamma}$ (Lugones et al. 1994). The corresponding energy density in the neutron phase is $\varepsilon_{n}=$ $n_{B} m_{n}+[1 /(\gamma-1)] K n_{B}^{\gamma}$, leading to $w_{n}=\varepsilon_{n}+P_{n}=n_{B} m_{n}+$ $[\gamma /(\gamma-1)] K n_{B}^{\gamma}$. We also assume that the formed quark bubble consists of $u$ - and $d$-quarks in the ratio $1: 2$; only later weak interactions may change the composition to an energetically more favorable state. The quarks' chemical potentials are related by $\mu_{d}=2^{1 / 3} \mu_{u}$, and, assuming chemical equilibrium across the phase boundary, we also have $\mu_{n}=\mu_{u}+2 \mu_{d}=$ $\left(1+2^{4 / 3}\right) \mu_{u}$ (Olesen \& Madsen 1994). Then the pressure in the quark phase is (assuming a simple bag model) $P_{q}=$ $\left[\left(\mu_{u}^{4}+\mu_{d}^{4}\right) / 4 \pi^{2}\right]-B$ and $\varepsilon_{q}=3 P_{q}+B$ (Cheng et al. 1998a), giving $w_{q}=\left[\left(\mu_{u}^{4}+\mu_{d}^{4}\right) / \pi^{2}\right]-3 B$. Hence, the enthalpy difference in the two phases can be approximated by

$$
\Delta w=\frac{\mu_{u}^{4}+\mu_{d}^{4}}{\pi^{2}}-3 B-n_{B} m_{n}-\frac{\gamma}{\gamma-1} K n_{B}^{\gamma} .
$$

Assuming that the energy flow is provided by the viscous effects only, one obtains for the prefactor the expression (Venugopalan \& Vischer 1994)

$$
k=\frac{2 \sigma}{(\Delta w)^{2} R_{c}^{3}}\left[\lambda_{n} T+2\left(\frac{4}{3} \eta_{n}+\xi_{n}\right)\right] .
$$

In the limit of zero baryon number, $\lambda_{n} \rightarrow 0$ and we obtain the result of Csernai \& Kapusta (1992a). If the matter is baryon-rich, but viscous damping is negligible, $\eta_{n}, \xi_{n} \rightarrow 0$, and we obtain the results of Langer \& Turski (1973) and Kawasaki (1975).

Therefore, the condition of the formation of a quark bubble is given by

$$
\begin{aligned}
& \frac{1}{\pi} \frac{n_{q} n_{n}}{(\Delta w)^{2} R_{c}} \sqrt{\frac{3 C^{3} T \sigma}{12 C^{3} \sqrt{1+b}-32 \pi\left(\rho_{q}-\rho_{n}\right)^{2} \sigma^{2}(1+\sqrt{1+b})^{3}}} \\
& \times\left[\lambda_{n} T+2\left(\frac{4}{3} \eta_{n}+\xi_{n}\right)\right] \exp \left\{-\frac{4 \pi \sigma^{3}}{3 C^{2} T} F(b)\right. \\
& \left.\times\left[1+4 \pi\left(\rho_{q}-\rho_{n}\right)^{2} \frac{\sigma^{2}}{C^{3}} G\left(b, b_{0}\right)\right]\right\} \Delta t \Delta V_{0} \geq 1 .
\end{aligned}
$$

The transport properties of dense matter have been intensively investigated in both high-energy physics and astrophysical frameworks (Flowers \& Itoh 1976, 1979, 1981; Hakim \& Mornas 1993). The shear viscosity $\eta$ and thermal conductivity $\lambda$ for neutron matter have been derived by Danielewitz (1984) from the Uhlenbeck-Uehling equation (see also Sawyer 1989; Cutler et al. 1990). They are given by $\eta=\left(16 \pi^{2}\right)^{-1}\left(p_{\mathrm{F}}^{5} / m_{n}^{2} \tilde{\sigma}_{1}\right) T^{-2}$ and $\lambda=5\left(p_{\mathrm{F}}^{3} / m_{n}^{2} \tilde{\sigma}_{2}\right) T^{-1} / 96$, where $p_{\mathrm{F}}$ is the Fermi momentum and $\tilde{\sigma}_{1}, \tilde{\sigma}_{2}$ are some quantities related to particle-particle cross sections, estimated in Danielewitz (1984). The contributions of the bulk viscosity and thermal conductivity are negligible, and the main contribution is from the shear viscosity, which is typically of the order of $\eta \sim 50 \mathrm{MeV} \mathrm{fm}^{-2}$ (Heiselberg 1995). But in the case of an ideal neutron gas, with zero viscosity, there will be no bubble growth, and in this case the transition from neutron matter to quark matter in astrophysical objects cannot take place.

Assuming that the energy flow does not vanish in the absence of any heat conduction or viscous damping, and considering that the viscous effects are small and can be neglected, the condition of the formation of a quark bubble inside the dense core of a neutron star becomes

$$
\begin{aligned}
& \frac{1}{\sqrt{2} \pi} \frac{n_{q} n_{n}}{\Delta w} \sqrt{\frac{3 C^{3} R_{c} T w_{n}}{12 C^{3} \sqrt{1+b}-32 \pi\left(\rho_{q}-\rho_{n}\right)^{2} \sigma^{2}(1+\sqrt{1+b})^{3}}} \\
& \times \exp \left\{-\frac{4 \pi \sigma^{3}}{3 C^{2} T} F(b)\left[1+4 \pi\left(\rho_{q}-\rho_{n}\right)^{2} \frac{\sigma^{2}}{C^{3}} G\left(b, b_{0}\right)\right]\right\} \\
& \times \Delta t V_{0} \geq 1 .
\end{aligned}
$$

For $r>R_{c}$ the time growth of the radius of the quark droplets can be described by the equation (Csernai \& Kapusta 1992a; Shukla et al. 2001)

$$
\frac{d r}{d t} \approx k R_{c}^{2} \frac{r-R_{c}}{r^{2}}
$$

with the general solution

$$
t \approx \frac{1}{k}\left[\frac{1}{2}\left(\frac{r}{R_{c}}\right)^{2}+\frac{r}{R_{c}}+\ln \frac{r-R_{c}}{R_{c}}-4\right],
$$

where we have used the initial condition $r(0)=2 R_{c}$. Therefore, for a neutron star with radius $R$, the time $t_{\text {conv }}$ required for the conversion of the whole star to a quark star can be obtained from

$$
t_{\mathrm{conv}} \approx \frac{1}{k}\left[\frac{1}{2}\left(\frac{R}{R_{c}}\right)^{2}+\frac{R}{R_{c}}+\ln \frac{R-R_{c}}{R_{c}}-4\right]
$$

Since $R \gg R_{c}$, with a very good approximation we find

$$
t_{\mathrm{conv}} \approx \frac{1}{2 k}\left(\frac{R}{R_{c}}\right)^{2}
$$

Neglecting the viscous effects, and by assuming again that the neutron matter is described by a polytropic EOS, while the quark phase obeys the simple bag model EOS, one obtains for the conversion time

$$
\begin{aligned}
t_{\mathrm{conv}} & \approx \frac{(\Delta w)^{2}}{\sqrt{8 \sigma R_{c} w_{n}}} R^{2} \\
& =\frac{\left\{\left[\left(\mu_{u}^{4}+\mu_{d}^{4}\right) / \pi^{2}\right]-3 B-n_{B} m_{n}-[\gamma /(\gamma-1)] K n_{B}^{\gamma}\right\}^{2}}{\sqrt{8 \sigma R_{c}\left\{n_{B} m_{n}+[\gamma /(\gamma-1)] K n_{B}^{\gamma}\right\}}} R^{2} .
\end{aligned}
$$


The volume $V(t)$ of the neutron matter converted to quark matter after a time interval $t<t_{\text {conv }}$ is given, in this simple model, by

$$
V(t) \approx \frac{2^{17 / 4} \pi\left(\sigma w_{n} R_{c}\right)^{3 / 4}}{3(\Delta w)^{3 / 2}} t^{3 / 2} .
$$

If the transition from neutron to quark phase is driven by viscous processes only, then

$t_{\mathrm{conv}} \approx$

$R_{c} \frac{\left\{\left(\mu_{u}^{4}+\mu_{d}^{4}\right) / \pi^{2}-3 B-n_{B} m_{n}-[\gamma /(\gamma-1)] K n_{B}^{\gamma}\right\}^{2}}{2 \sigma\left\{\lambda_{n} T+2\left[(4 / 3) \eta_{n}+\xi_{n}\right]\right\}} R^{2}$,

and

$$
\begin{gathered}
V(t) \approx \frac{2^{5} \pi \sigma^{3 / 2}}{3(\Delta w)^{3} R_{c}^{3 / 2}}\left[\lambda_{n} T+2\left(\frac{4}{3} \eta_{n}+\xi_{n}\right)\right]^{3 / 2} t^{3 / 2} . \\
\text { 4. ENDOTHERMIC NUCLEATION OF QUARK } \\
\text { MATTER BUBBLES IN THE CORE OF } \\
\text { ROTATING NEUTRON STARS }
\end{gathered}
$$

In the previous sections we have considered the process of nucleation of quark bubbles in neutron star cores, and we have derived the conditions for quark matter formation in an astrophysical context. In the present section we apply the results previously obtained to analyze the possibility of the transition from neutron to quark phase during the spinning down of a rapidly rotating neutron star. In this case the rotational energy of the star can be used to trigger the phase transition.

The minimum energy density required to create a quark bubble is

$$
\begin{aligned}
u_{q} & =\frac{3 W_{c}}{4 \pi R_{c}^{3}} \\
& =\frac{C}{(1+\sqrt{1+b})^{3}} F(b)\left[1+\frac{24 \pi}{5}\left(\rho_{q}-\rho_{n}\right)^{2} \frac{\sigma^{2}}{C^{3}} G(b)\right] .
\end{aligned}
$$

An estimation of equation (39) for $C \approx 20 \mathrm{MeV} \mathrm{fm}{ }^{-3}$, $\gamma \approx 18 \mathrm{MeV} \mathrm{fm}^{-1}, \sigma \approx(75 \mathrm{MeV})^{3}, \rho_{q} \approx-0.4 e \mathrm{fm}^{-3}$, and $\rho_{n} \approx 0$ gives $u_{q} \approx 3.76 \times 10^{34} \mathrm{ergs} \mathrm{cm}^{-3}$.

We consider that the evolution of the rotating neutron star can be approximated by a sequence of MacLaurin spheroids (Chandrasekhar 1986; Cheng et al. 1992). The mass of the star is denoted by $M$ and the major and minor axis of the star by $a$ and $c$ (equatorial and polar radius). Then the eccentricity of the star is defined according to $e=\left[1-(c / a)^{2}\right]^{1 / 2}$. Let $\rho=$ const denote the density of the star, corresponding to a given value of the angular velocity $\Omega$ and of the equatorial radius $a$.

Hence, the basic equations describing the mass, hydrostatic equilibrium, and gravitational energy $E_{\mathrm{gr}}$ of the rotating star are (Chandrasekhar 1986)

$$
\begin{gathered}
M=\frac{4 \pi}{3} a^{3} \rho\left(1-e^{2}\right)^{1 / 2}, \quad \Omega^{2}=2 \pi G \rho f(e), \\
E_{\mathrm{gr}}=-\frac{3}{5} \frac{G M^{2}}{a} \frac{\arcsin e}{e},
\end{gathered}
$$

where

$$
f(e)=\frac{\left(1-e^{2}\right)^{1 / 2}}{e^{3}}\left(3-2 e^{2}\right) \arcsin e-\frac{3\left(1-e^{2}\right)}{e^{2}} .
$$

Assuming that the total mass of the star is a constant, one obtains the following relation between the variation $\Delta a, \Delta \Omega$, $\Delta \rho$, and $\Delta e$ of the equatorial radius, angular velocity, density, and eccentricity, respectively:

$$
\frac{3}{a} \frac{\Delta a}{\Delta \Omega}+\frac{1}{\rho} \frac{\Delta \rho}{\Delta \Omega}=\frac{e}{1-e^{2}} \frac{\Delta e}{\Delta \Omega} .
$$

The variation of the eccentricity with respect to the angular velocity is given by

$$
\frac{\Delta e}{\Delta \Omega}=\frac{\Omega-\pi G f(e)(\Delta \rho / \Delta \Omega)}{\pi G \rho g(e)},
$$

where

$$
\begin{aligned}
g(e) & =\frac{\Delta f(e)}{\Delta e} \approx \frac{d f(e)}{d e} \\
& =\frac{2\left(9-2 e^{2}\right) \sqrt{1-e^{2}}+\left(8 e^{2}-9\right) \arcsin e}{e^{4} \sqrt{1-e^{2}}} .
\end{aligned}
$$

With the use of the above equations the variation of the gravitational energy of the neutron star, corresponding to a simultaneous change in equatorial radius, angular velocity, and density, is given by

$\Delta E_{\mathrm{gr}}(a, \rho, \Omega)=\frac{3}{5} \frac{G M^{2}}{a}\left[A(e) \frac{\Omega}{\pi G \rho}-B(e) \frac{1}{\rho} \frac{\Delta \rho}{\Delta \Omega}\right] \Delta \Omega$,

where we denote

$$
\begin{gathered}
h(e)=\frac{1}{3} \frac{e}{1-e^{2}}-\frac{1}{e \sqrt{1-e^{2}}}+\frac{\arcsin e}{e^{2}}, \\
A(e)=\frac{h(e)}{g(e)}, \quad B(e)=\frac{h(e) f(e)}{g(e)}+\frac{1}{3} .
\end{gathered}
$$

The gravitational energy density released as the result of the slowing down of the neutron star can trigger the endothermic phase transition at the center of the star. Inside a sphere of radius $R_{q}$ the gravitational energy density is

$u_{\mathrm{gr}}(a, \rho, \Omega)=\frac{9}{20 \pi} \frac{G M^{2}}{a} R_{q}^{-3}\left[A(e) \frac{\Omega}{\pi G \rho}-B(e) \frac{1}{\rho} \frac{\Delta \rho}{\Delta \Omega}\right] \Delta \Omega$.

This energy should be greater than or equal to the minimum energy necessary for the formation of a quark bubble, given by equation (39). Therefore, the gravitational energy converts to the quark phase the neutron matter inside a sphere of radius

$$
\begin{aligned}
R_{q}(a, \rho, \Omega)= & 2.88\left(\frac{M}{M_{\odot}}\right)^{2 / 3}\left(\frac{a}{10^{6}}\right)^{-1 / 3} \\
& \times\left[A(e) \frac{\Omega}{\pi G \rho}-B(e) \frac{1}{\rho} \frac{\Delta \rho}{\Delta \Omega}\right]^{1 / 3} \\
& \times \frac{(1+\sqrt{1+b}) C^{-1 / 3}}{\left[2+2(1+b)^{3 / 2}+3 b\right]^{1 / 3}}(\Delta \Omega)^{1 / 3} \times 10^{6} \mathrm{~cm} .
\end{aligned}
$$


In order for the whole neutron star to be converted to a quark star, the condition $R_{q}(\rho, \Omega)=a(\rho, \Omega)$ must hold. On the other hand, for typical neutron star densities of the order of $10^{14}-10^{15} \mathrm{~g} \mathrm{~cm}^{-3}$, the term $A(e) \Omega / \pi G \rho$ is much smaller than $B(e)(\Delta \rho / \Delta \Omega) / \rho$,

$$
A(e) \frac{\Omega}{\pi G \rho} \ll B(e) \frac{1}{\rho} \frac{\Delta \rho}{\Delta \Omega} .
$$

For small eccentricities $e$ the function $B(e)$ can be approximated by $\frac{1}{3}$.

Therefore, we obtain the following condition for the relative change in the central density, necessary to convert a neutron star to a strange star, due to the endothermic gravitational energy transfer in the whole volume of the star:

$$
\frac{\Delta \rho}{\rho} \approx 0.125 \frac{C\left[2+2(1+b)^{3 / 2}+3 b\right]}{(1+\sqrt{1+b})^{3}}\left(\frac{M}{M_{\odot}}\right)^{-2 / 3} .
$$

For a neutron star of around two solar masses, $M \approx 2 M_{\odot}$, and by assuming $C \approx 20 \mathrm{MeV}$ and $b \approx 6$, we obtain $\Delta \rho / \rho \approx 1.85$.

Of course, this result is strongly dependent on the precise numerical values of $C$ and $b$, which are generally poorly known; $C$ is also a neutron matter EOS-dependent parameter. For example, assuming for $C$ a value of $10 \mathrm{MeV}$ will lead to $\Delta \rho / \rho \approx 0.92$. An increase in the mass $M$ of the star will also decrease the value of the relative change in the density of the star necessary to convert the neutron star.

At present, we have very little observational knowledge regarding how fast newborn neutron stars can rotate. The most well-known young pulsar is the Crab pulsar, which was born with a rotational period of about $20 \mathrm{~ms}$ (Manchester \& Taylor 1977). If so, during its lifetime the pulsar's central density only increases by about $\Delta \rho_{c} / \rho_{c} \approx 0.001$ (Ma \& Xie 1996). Currently, the fastest rotating pulsar known is PSR 1937+214, which has a period of $1.55 \mathrm{~ms}$ or $\Omega \approx 4000 \mathrm{~s}^{-1}$, but it has weak magnetic field, and it has been suggested that it is spun up by accretion (Alpar et al. 1982). However, it has been suggested that some rapidly spinning millisecond pulsars are be born by accretion-induced collapse from white dwarfs (Arons 1983). Therefore, it cannot be ruled out that some pulsars may be born with millisecond periods and strong magnetic field.

The theoretical investigation of rotating general relativistic objects performed by Cook et al. (1994) shows that for some realistic EOSs of neutron matter, this variation of the central density can be achieved during the complete spin-down of the star (see Table 1).

The central density increase due to the spin-down can be easily realized in a short time interval for a special class of stellar-type objects, called magnetars. Magnetars are compact objects with superstrong magnetic fields of the order $B \approx$ $10^{15} \mathrm{G}$ or even higher (Duncan \& Thompson 1992; Paczynski 1992; Thompson \& Duncan 1995, 1996). It is now believed that soft gamma repeaters (SGRs) - a small class (four confirmed and one candidate) of high-energy transients discovered through their emission of bright X-ray/gamma-ray bursts, which repeat on timescales of seconds to years - are magnetars. There is evidence that the giant SGR flares involve the cooling of a confined $e^{ \pm}$-photon plasma in an ultrastrong magnetic field (Lyubarsky et al. 2002). Some authors (Cheng \& Dai 1998; Usov 2001), motivated in part by the superEddington luminosities of the SGRs' giant flares, have suggested that they are strange stars. The magnetars differ from the
TABLE 1

Variation of the Central Density of the Rapidly Rotating Neutron

\begin{tabular}{|c|c|c|c|c|}
\hline EOS & $\begin{array}{l}M_{\text {stat }} \\
\left(M_{\odot}\right)\end{array}$ & $\begin{array}{l}R_{\text {stat }} \\
(\mathrm{km})\end{array}$ & $\begin{array}{c}\Delta \Omega \\
\left(\mathrm{s}^{-1}\right)\end{array}$ & $\Delta \rho_{c} / \rho_{c}$ \\
\hline A & 1.6551 & 8.368 & 10011 & 1.26 \\
\hline 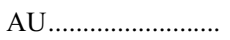 & 2.1335 & 9.411 & 10587 & 0.97 \\
\hline FPS ........................... & 1.7995 & 9.281 & 8874.9 & 0.91 \\
\hline 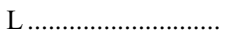 & 2.7002 & 13.7 & 6482.9 & 1.244 \\
\hline 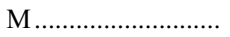 & 1.8045 & 11.6 & 4437 & 4.189 \\
\hline
\end{tabular}
Star for Different Equations of State

NoTE.-Variation of the central density of the rapidly rotating neutron star for different EOSs (Cook et al. 1994): A (Pandharipande 1971), AU (Wiringa et al. 1988), FPS (Lorentz et al. 1993), L (Pandharipande et al. 1976), and M (Pandharipande \& Smith 1975).

canonical pulsars (with low magnetic fields of the order of $\left.B \approx 10^{11}-10^{13} \mathrm{G}\right)$ in the sense that they spin down much more rapidly.

If we assume that the spin-down of the magnetar is completely determined by the torque of its relativistic wind emission, generated via the magnetic dipole radiation, then the time variation of the angular velocity $\Omega$ of the star is given by Shapiro \& Teukolsky (1983),

$$
I \dot{\Omega}=-\frac{2}{3} \frac{\mu^{2} \Omega^{3}}{c^{3}},
$$

where $I$ is the moment of inertia of the star and $\mu=R^{3} B$ is the magnetic dipole moment. When the star is born with a spin period much shorter than the observed one, the age of the star is the spin-down age $\tau_{\text {sd }}=P / \dot{P}$, where $P$ and $\dot{P}$ are the present spin period and its time derivative, respectively. If the spindown is entirely due to the magnetic dipole radiation, we obtain

$$
\tau_{\text {sd }}=\frac{\Omega}{\dot{\Omega}}=\frac{3}{2} \frac{I c^{3}}{B^{2} R^{6} \Omega^{2}} .
$$

By adopting for the moment of inertia and the radius of the magnetar the typical values $I=10^{45} \mathrm{~g} \mathrm{~cm}^{2}$ and $R=10^{6} \mathrm{~cm}$, and by assuming that the star was born with a magnetic field of the order of $B \approx 10^{15} \mathrm{G}$ and with an angular velocity of $\Omega_{0}=6000 \mathrm{rad} \mathrm{s}^{-1}$, the spin-down age is given by $\tau_{\mathrm{sd}} \approx$ $1125 \mathrm{~s}$.

From equation (52) it follows that the decay law of the angular velocity $\Omega$ of the star is of the form

$$
\Omega=\frac{\Omega_{0}}{\sqrt{(4 / 3)\left(B^{2} \Omega_{0}^{2} R^{6} / c^{3} I\right) t+1}},
$$

where $\Omega_{0}$ is the initial angular velocity of the star.

Hence, in a time interval of around $t \approx 10^{6} \mathrm{~s}$ and for a magnetic field $B=10^{15} \mathrm{G}$ the angular velocity of the star decreases from $\Omega_{0}=6000 \mathrm{rad} \mathrm{s}^{-1}$ to $\Omega=142 \mathrm{rad} \mathrm{s}^{-1}$. For an initial angular velocity of the order of $\Omega=8000 \mathrm{rad} \mathrm{s}^{-1}$ and for a magnetic field of the order of $B \approx 10^{16} \mathrm{G}, \tau_{\mathrm{sd}} \approx 5 \mathrm{~s}$. In a time interval of around $t \approx 600 \mathrm{~s}$ the angular velocity of the star decreases to $\Omega \approx 579 \mathrm{rad} \mathrm{s}^{-1}$.

Therefore, in a magnetar density changes, due to the spindown, take place in a short interval of time. Hence, they can provide enough energy to trigger spontaneous nucleation to the quark phase, once the central density of the star goes above the critical density. 
The nucleation of quark droplets in the presence of strong magnetic fields essentially depends on the strength of the magnetic field. The energy of a quantum particle changes significantly if the magnetic field is of the order or exceeds the critical value $B_{c}=m^{2} c^{3} / e \hbar$, where $m$ is the mass of the particle. For electrons the value of the critical field is $B_{c}^{(e)}=4.4 \times$ $10^{13} \mathrm{G}$. In order to calculate the value of the critical magnetic field for quarks, we have to use the estimations for the quark masses. However, there are many uncertainties in the numerical values of the quark masses, which play an essential role in the calculation of the critical magnetic field and are generally poorly known. In the case of free quarks, the current quark mass is considered to be in the range of $m_{q} \sim 5-10 \mathrm{MeV}$. Then the corresponding critical magnetic field is $B_{c}^{(q)} \sim$ $(1-2) \times 10^{2} B_{c}^{(e)} \approx(4.4-8.8) \times 10^{15} \mathrm{G}$. For the current quark mass, this is the typical strength of the magnetic field at which the cyclotron lines begin to occur. In this limit the cyclotron quantum is of the order of or greater than its rest energy. This is equivalent to the requirement that the de Broglie wavelength is of the order of or greater than the Larmor radius of the particle in the magnetic field (Chakrabarty 1995). However, from the physical point of view a better description of the quark mass can be obtained by using, instead of the current quark mass, the effective or constitute quark mass, which takes into account the effects of the strong interactions, and which is of the order $m_{q} \sim 100-300 \mathrm{MeV}$ (Cheng et al. 1998a). The critical magnetic field corresponding to the effective quark mass could be as high as $B_{c}^{(q)} \sim 10^{17}-10^{18} \mathrm{G}$.

The study of the behavior of the surface and curvature energy terms in strong magnetic fields has shown that both quantities diverge for $B>B_{c}^{(q)}$, with the curvature term diverging much faster. Consequently, in the presence of strong magnetic fields the rate of stable quark droplet formation per unit volume $I \sim T^{4} \exp (-W / T)$ tends to zero, $I \rightarrow 0$, and therefore there cannot be any thermal nucleation of quark droplets in neutron star cores (Chakrabarty 1995, 1996).

The value of $B$ inside a magnetar is not known, and it is not certain if the interior magnetic field is much stronger than the surface field. If in the interior of the magnetar the value of the magnetic field can exceed the critical value $B_{c}^{(q)} \sim 5 \times 10^{15} \mathrm{G}$, which represents the most conservative estimation of the critical magnetic field, corresponding to the free quark mass $m=5 \mathrm{MeV}$, then the rate of droplet formation can be considerably reduced and the transition from neutron matter to quark matter could not take place.

On the other hand, by taking into account that the physical mass of the quark is the effective mass, the corresponding critical magnetic field could be of the order $B_{c}^{(q)} \sim 10^{17}-10^{18}$ $\mathrm{G}$, a value that could be higher than the magnitude of the magnetic field inside the magnetars. For magnetic fields smaller than $B_{c}^{(q)}$, the endothermic nucleation process can take place in a short time interval during the spin-down of the magnetar.

\section{DISCUSSIONS AND FINAL REMARKS}

In this paper we have considered a kinetic theory of strange matter nucleation in neutron stars. We derived general necessary conditions for neutron to quark matter conversion. There have been several assumptions made in the derivation of the results, mostly related to the form of the growth speed of the bubble. The result is valid only if nonlinear effects can be ignored, and the linearized hydrodynamic equations are applicable. Furthermore, we have assumed that the radii of the bubbles are larger than the correlation length and that heating due to dissipation is slow, causing the temperature to vary slowly across the bubble wall. We also considered that the phase transition is strongly first order, releasing considerable amounts of heat.

The growth of the critical size quark bubbles nucleated in the first-order transition in the neutron star core is governed by the dynamical prefactor $k$. There are several expressions for the prefactor obtained in the physical literature. In the CsernaiKapusta approach (Csernai \& Kapusta 1992a), the prefactor essentially depends on the dissipative properties (shear, bulk viscosity, and thermal conductivity) of neutron matter. Therefore, in a nonviscous medium with $\xi_{n}=\eta_{n}=\lambda_{n}=0$, the growth of the quark bubbles cannot take place. The main physical assumption is that the energy flow is provided by the viscous effects only.

On the contrary, one can argue that the energy flow does not vanish in the absence of heat conduction or viscous damping (Ruggeri \& Friedman 1996; Shukla et al. 2001) . In this case, and in the limit of zero viscosity, the prefactor depends only on two scale parameters, the correlation length $\zeta$ and the critical radius of the quark bubble $R_{c}$. For a viscous medium the prefactor can simply be written as the sum of the viscous and nonviscous terms, with the viscous term not affecting the growth process significantly. The previous (viscous) results can be reobtained by using some assumptions for the velocity of sound in the medium around the saddle configuration.

The condition for the formation of at least a quark bubble in the neutron matter as a result of the thermal fluctuation is given by equation (29). The condition includes the cases of both viscous and ideal neutron matter. It is extremely sensitive with respect to the numerical values of the microscopic parameters characterizing the quark bubble, such as the surface tension $\sigma$, the bag constant $B$, or the curvature coefficient $\gamma$. In discussing the astrophysical implications of equation (29), we consider separately the two limiting cases, corresponding to viscous and nonviscous neutron matter, respectively.

In the case of ideal neutron matter, the condition of the formation of a quark bubble is given by equation (30). This equation determines at which temperature the phase transition takes place. For example, in the case of the Walecka mean field equation of state (Walecka 1975), with $K=15758$ and $\gamma=4.95$ (Lugones et al. 1994), the temperature $T_{c}$ necessary for the formation of a stable quark bubble in a second $(\Delta t=1 \mathrm{~s})$, in a volume $\Delta V_{0}$ with radius $\Delta R_{0}=1 \mathrm{~km}\left(\Delta V_{0}=4 \pi / 3 \mathrm{~km}^{3}\right)$, and at the center of the neutron star is around $T_{c}=12-$ $13 \mathrm{MeV}$, for $\sigma=(85 \mathrm{MeV})^{3}$. Here we have also assumed the standard values for the quark matter chemical potential and for the bag constant, $\mu_{u}=\mu_{d}=280 \mathrm{MeV}$ and $B=60 \mathrm{MeV} \mathrm{fm}^{-3}$ (Cheng et al. 1998a). Since the temperature of a newborn neutron star is around $10 \mathrm{MeV}$ (Olesen \& Madsen 1994), this value of the surface tension seems to rule out, for the given equation of state, the possibility of quark nuclei forming in the neutron matter. However, a small variation in the value of $\sigma$, $\sigma=(75 \mathrm{MeV})^{3}$, reduces the temperature for quark nuclei formation to $T_{c}=4-6 \mathrm{MeV}$, a value that does not exclude the possibility that quark nuclei formation can be initiated in the early stages of neutron star evolution. Smaller values of $\sigma$, of the order of $\sigma=(50 \mathrm{MeV})^{3}$, can lower the phase transition temperature even more, making it possible even for cold neutron stars. The transition temperature is relatively insensitive to the details of the equation of state of the dense neutron matter.

If the temperature at which the phase transition is initiated is strongly dependent on the microscopic model adopted for describing the neutron matter properties, the total transition 
time of the neutron star to a quark star, given by equation (35), is much less dependent on the details of the equation of state or the exact numerical values of the surface tension or curvature of the bubble. Generally, for a neutron star with radius $R=12-15 \mathrm{~km}$, the conversion time is very long, of the order of $10^{6} \mathrm{yr}$ for $\sigma \approx(75 \mathrm{MeV})^{3}$. For $\sigma \approx(50 \mathrm{MeV})^{3}$ or even lower, the conversion time could have values of the order of $10^{7} \mathrm{yr}$, which are still of the same order of magnitude as the lifetime of a neutron star $\left(10^{7} \mathrm{yr}\right)$. Hence, in the present approach, which is limited to the consideration of linear effects only in the hydrodynamic description of the first-order phase transitions for an ideal neutron fluid, the growth of the quark nuclei is very slow. Therefore, the results of the present analysis suggest that even though quark nuclei could appear inside the neutron star at a very early stage, the transition to a pure quark phase ends only in the final stage of its evolution.

If the neutron matter-quark matter phase transition is mainly driven by viscous processes, as in the CK scenario, then the time necessary for a neutron star to convert to a quark star follows from equations (28) and (34). In order to numerically estimate the conversion time, we need an estimation of viscosity and conduction coefficients of nuclear matter. We also consider two limiting cases for the phase transition. Below the critical density $\rho=\rho_{c} \approx 10^{14} \mathrm{~g} \mathrm{~cm}^{-3}$, cold neutron matter consists of two distinct phases. At low density, neutron star matter consists of nuclei that form a solid lattice for temperatures below the melting temperature and a sea of relativistic electrons. When the density reaches $\rho \approx 10^{11} \mathrm{~g} \mathrm{~cm}^{-3}$, neutrons begin to "drip" from the neutron-rich nuclei and then, in addition to the relativistic electrons, there is a sea of nonrelativistic neutrons. At the critical density the neutronrich nuclei dissolve, leaving seas of degenerate neutrons, protons, and electrons. The neutrons in cold neutron star matter are quite likely superfluid, and therefore the transport coefficients are dominated by electrons, with the motion of electrons determined by electron-electron and electron-proton scattering (Flowers \& Itoh 1979). As a result, the viscosity is much lower, but the thermal conductivity is only slightly smaller as compared to the case in which both neutrons and protons form normal fluids (Flowers \& Itoh 1979). Hence, we can neglect the viscosity coefficients with respect to the thermal conductivity and approximate $\lambda_{n}$ by $\lambda_{n} \approx \lambda_{0} \rho_{14} / T_{8}$, with $\lambda_{0} \approx 10^{23}$ (Flowers \& Itoh 1979). A slightly different expression for the leading term in thermal conductivity has been proposed in Danielewitz (1984), $\lambda_{n} T \approx \lambda_{0}\left(n / n_{0}\right)^{1 / 4}$, with $\lambda_{0}=0.15 \mathrm{fm}^{-3}$ and $n_{0}=0.145 \mathrm{fm}^{-3}$. Hence, by using this last expression, we obtain for $t_{\text {conv }}$

$$
t_{\text {conv }} \approx R_{c} \frac{(\Delta w)^{2}}{4 \sigma \lambda_{0}\left(n / n_{0}\right)^{1 / 4}} R^{2} .
$$

The transition time is independent of the temperature of the neutron matter. It depends only on the microscopic properties of the quark bubble (surface tension and curvature coefficient), density, and the equation of state of the matter inside the star. For $\mu_{u}=\mu_{d}=280 \mathrm{MeV}, B=60 \mathrm{MeV} \mathrm{fm}^{-3}$, and $\sigma \approx$ $(75 \mathrm{MeV})^{3}$ equation (55) gives, for a neutron star with radius $R=12 \mathrm{~km}$ and for several equations of state, values of the conversion time of the order $t_{\text {conv }} \approx 10^{11} \mathrm{yr}$.

In the case of young and hot neutron stars, the main dissipative mechanism that could drive the phase transition is the shear viscosity of the neutron matter. The temperature dependence of the shear viscosity coefficient can be approximated by $\eta_{n} \approx\left(\eta_{0} / T^{2}\right)\left(n / n_{0}\right)^{2}$, with $\eta_{0}=1700 \mathrm{MeV}^{3} \mathrm{fm}^{-2}$
(Danielewitz 1984). Consequently, by neglecting the heat conduction and the bulk viscosity, the conversion time becomes

$$
t_{\mathrm{conv}} \approx \frac{3}{32} R_{c} \frac{(\Delta w)^{2}}{\sigma \eta_{n}} R^{2} \approx \frac{3}{32} R_{c} \frac{(\Delta w)^{2}}{\sigma \eta_{0}\left(n / n_{0}\right)^{2}} T^{2} R^{2} .
$$

In small temperatures, the shear viscosity becomes very large. Hence, for a shear viscosity-driven phase transition the conversion time could be very small.

In Figure 1 we have represented $t_{\text {conv }}$ given by equation (56) as a function of temperature, for different equations of state of neutron matter. We have considered four equations of state, namely, the equation of state (EOS) of the free neutron gas (Shapiro \& Teukolsky 1983), the Bethe-Johnson EOS (Shapiro \& Teukolsky 1983), the Lattimer-Ravenhall EOS (Lattimer \& Ravenhall 1978), and the Walecka EOS (Walecka 1975). In all cases we calculated the conversion time for a neutron star with radius $R=10 \mathrm{~km}$.

As one can see from the figure, in this case the conversion time is much shorter as compared to the ideal neutron matter case or thermal conduction-driven phase transition. Time $t_{\text {conv }}$ is rapidly decreasing with the temperature. For a neutron star with rapidly cooling processes (e.g., direct Urca process, kaon condensation), the stellar temperature can decrease to less than $10^{-2} \mathrm{MeV}$ in a timescale of $10^{-4} \mathrm{yr}$ (Tsuruta 1998). According to Figure 1, the conversion can take place on a timescale less than $10^{-3} \mathrm{yr}$ if the spin-down timescale is less than this timescale. This can be the case if this neutron star is a magnetar. We should point out that the interiors of magnetars are not quantum liquid, i.e., superfluid or superconducting, because the strong magnetic field can destroy the nucleon cooper pairs (Chau et al. 1992).

On the other hand, if the neutrons and protons are superfluid, then the transport properties are completely determined by electron-electron scattering. In fact, in this case the viscosity of the nuclear fluid is given by $\eta^{-1}=\left(15 \pi^{4} \alpha^{2} / 2 p_{e}^{5}\right)\left(2 p_{e} / k_{\mathrm{TF}}\right)$ $\left[5 / 2+3\left(m_{e} / p_{e}\right)^{2}+\left(m_{e} / p_{e}\right)^{4}\right] T^{2}$ (Flowers \& Itoh 1976), where $\alpha$ is the fine-structure constant, $k_{\mathrm{TF}}=\left(4 \alpha p_{e} \epsilon_{e} / \pi\right)^{1 / 2}$ is the Thomas-Fermi wavevector, and $m_{e}, p_{e}$, and $\epsilon_{e}$ are the electron mass, momentum, and energy, respectively.

We assume that the electron gas inside the neutron star is extreme relativistic and strongly degenerate. Then it follows that $p_{e} \approx p_{\mathrm{F}}=\left(3 n_{e} / 8 \pi\right)^{1 / 3}$ (Shapiro \& Teukolsky 1983), with $p_{\mathrm{F}}$ the Fermi momentum and $n_{e}$ the electron number density. Since $n_{e}$ can be related to the total particle number $n$ by means of the relation $n_{e}=Y_{e} n$, with $Y_{e}$ the mean number of electrons per baryon, it follows that $p_{e} \approx\left(3 Y_{e} n_{0} / 8 \pi\right)^{1 / 3}\left(n / n_{0}\right)^{1 / 3}$. Hence, we obtain for the shear viscosity coefficient of the nuclear matter

$$
\begin{aligned}
\eta^{-1} \approx & \frac{15}{2} \frac{8^{5 / 3} \pi^{37 / 6} \alpha^{3 / 2}}{\left(3 Y_{e} n_{0}\right)^{5 / 3}\left(n / n_{0}\right)^{5 / 3}}\left\{\frac{5}{2}+3\left[\frac{m_{e}(8 \pi)^{1 / 3}}{\left(3 Y_{e} n_{0}\right)^{1 / 3}\left(n / n_{0}\right)^{1 / 3}}\right]^{2}\right. \\
& \left.+\left[\frac{m_{e}(8 \pi)^{1 / 3}}{\left(3 Y_{e} n_{0}\right)^{1 / 3}\left(n / n_{0}\right)^{1 / 3}}\right]^{4}\right\} T^{2} .
\end{aligned}
$$

The variation of the conversion time, as a function of temperature, is represented, for a superfluid neutron and proton core of the star with $Y_{e}=0.1$, in Figure 2.

In this case, since the viscosity of the superfluid nuclear matter is much smaller, the conversion time is longer than for a nuclear fluid dominated by the neutron viscosity. However, 


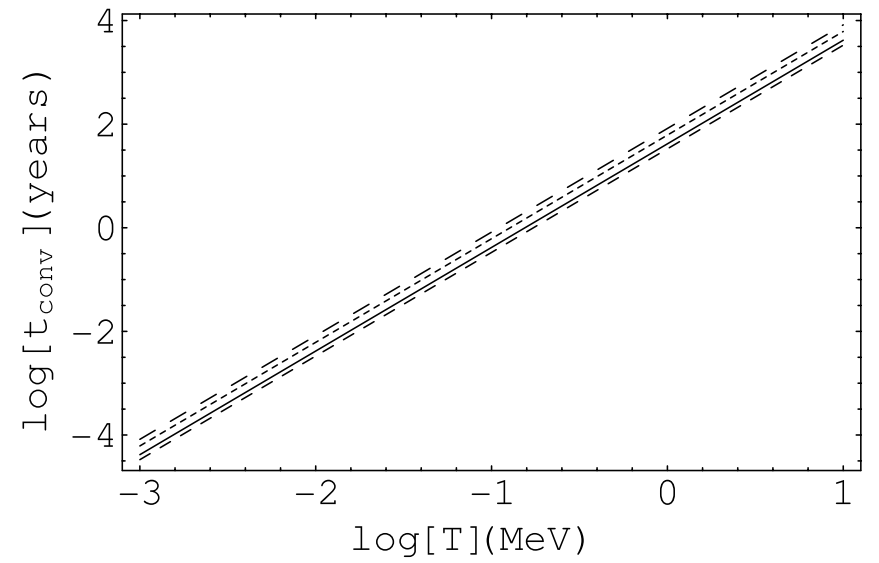

FIG. 1.-Variation of the conversion time $t_{\text {conv }}$ for a neutron star with radius $R=10 \mathrm{~km}$, for different EOSs of the neutron matter: free neutron EOS (solid curve), Bethe-Johnson EOS (dotted curve), Lattimer-Ravenhall EOS (shortdashed curve), and Walecka EOS (long-dashed curve).

in this case the phase transition from neutron matter to quark matter can also take place at very low temperatures. Consequently, a very short $t_{\text {conv }}$, of the order of hours or a few days, is also allowed in the framework of this model.

It is widely accepted that at asymptotic densities the ground state of QCD with $m_{s}=0$ is the color-flavor locked (CFL) phase (Alford et al. 1998; Rajagopal \& Wilczek 2001). In this phase, color gauge symmetry is completely broken, as are both chiral symmetry and baryon number. The effective coupling is weak and the low-energy properties can be determined by using methods from the theory of superconductivity. For large gaps $(\Delta \sim 100 \mathrm{MeV})$, the CFL phase is rigorously electrically neutral, despite the unequal quark masses and even in the presence of electron chemical potential. The transition from nuclear matter to quark matter via bubble nucleation can be greatly simplified if the transition occurs directly to quark matter in the CFL phase (Rajagopal \& Wilczek 2001). For a given baryonic chemical potential $\bar{\mu}$, electrically neutral nuclear matter and electrically neutral quark matter have different values of the electronic chemical potential $\mu_{e}$. Since $\mu_{e}$ must be continuous across any interface, a mixed phase region is formed, within which positively charged nuclear matter and negatively charged quark matter with the same $\mu_{e}$ coexist at any given radius. The growth of the quark droplets is at the expense of the nuclear matter. However, if the quark matter is in the CFL phase, an interface between bulk nuclear matter with nonzero electron number $N_{e} \neq 0$ and CFL quark matter with $N_{e}=0$ may be stable, as long as $\mu_{e}$ satisfies the condition $\left|m_{s}^{2} / 4 \bar{\mu}-\delta \mu\right|<\Delta / \sqrt{2}$, where $\delta \mu$ is the variation of the chemical potential (Rajagopal \& Wilczek 2001). Therefore, the existence of the CFL phase supports the idea that in

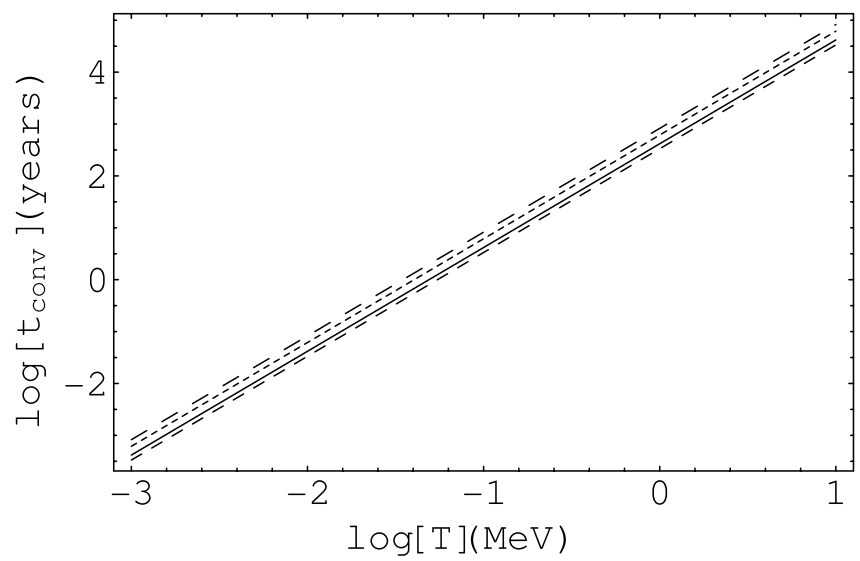

FIG. 2.-Variation of the conversion time $t_{\text {conv }}$ for a neutron star with radius $R=10 \mathrm{~km}$ to a quark star, in the case of the presence of a superfluid core, for different EOSs of the neutron matter: free neutron EOS (solid curve), BetheJohnson EOS (dotted curve), Lattimer-Ravenhall EOS (short-dashed curve), and Walecka EOS (long-dashed curve).

neutron stars quark matter and baryonic matter may be continuously connected. Then the growth of the interface between the neutron and CFL quark phase could be the result of exterior energy absorption, via an endothermic mechanism.

In summary, by using the kinetic nucleation theory, we have considered the possibility that the neutron matter-quark matter phase transition could be triggered by the gravitational energy released during the spin-down of a pulsar. This process could take place in a very short time interval, of the order of a few tens of seconds, in a class of dense compact objects, with very high magnetic fields, called magnetars. However, the percentage of neutron star matter that can be converted into quark matter depends on the initial period, the neutron star mass, and the equation of state of the neutron and quark phases. An important and open issue is how we could determine if such a process is taking place or has already taken place in some pulsars or magnetars, as well as what the observational signatures of the endothermic neutron matter-quark matter transition are. In particular, it would be interesting to know if such a process could be a possible gamma-ray burst mechanism, as suggested by Berezhiani et al. (2003). All of these issues will be considered in a subsequent paper (P. S. Tang et al. 2004, in preparation).

This work is supported by an RGC grant of the Hong Kong government of the SAR of China. The authors would like to thank the anonymous referee for comments that helped to improve the manuscript.
Alcock, C., Farhi, E., \& Olinto, A. 1986, ApJ, 310, 261

Alcock, C., \& Olinto, A. 1989, Phys. Rev. D, 39, 1233

Alford, M., Rajagopal, K., \& Wilczek, F. 1998, Phys. Lett. B, 422, 247

Alpar, M. A., Cheng, A. F., Ruderman, M. A., \& Shaham, J. 1982, Nature, 300,728

Arons, J. 1983, Nature, 302, 301

Berezhiani, Z., Bombaci, I., Drago, A., Frontera, F., \& Lavagno, A. 2003, ApJ, 586, 1250

Bodmer, A. R. 1971, Phys. Rev. D, 4, 1601

Caldwell, R. R., \& Friedman, J. R. 1991, Phys. Lett. B, 264, 143

Chakrabarty, S. 1993, Phys. Rev. D, 48, 1409

. 1995, Phys. Rev. D, 51, 4591

\section{REFERENCES}

Chakrabarty, S. 1996, Phys. Rev. D, 54, 1306

Chakrabarty, S., \& Sahu, P. K. 1996, Phys. Rev. D, 53, 4687

Chandrasekhar, S. 1986, Ellipsoidal Figures of Equilibrium (New York: Dover)

Chau, H. F., Cheng, K. S., \& Ding, K. Y. 1992, ApJ, 399, 213

Cheng, K. S., Chau, W. Y., Zhang, J. L., \& Chau, H. F. 1992, ApJ, 396, 135

Cheng, K. S., \& Dai, Z. G. 1996, Phys. Rev. Lett., 77, 1210 1998, Phys. Rev. Lett., 80, 18

Cheng, K. S., Dai, Z. G., \& Lu, T. 1998a, Int. J. Mod. Phys. D, 7, 139

Cheng, K. S., Dai, Z. G., Wei, D. M., \& Lu, T. 1998b, Science, 280, 407

Cheng, K. S., \& Harko, T. 2000, Phys. Rev. D, 30, 083001 2003, ApJ, 596, 451

Cook, G. B., Shapiro, S. L., \& Teukolsky, S. A. 1994, ApJ, 424, 823 
Csernai, L. P., \& Kapusta, J. I. 1992a, Phys. Rev. D, 46, 1379 1992b, Phys. Rev. Lett., 69, 737

Cutler, C., Lindblom, L., \& Splinter, R. J. 1990, ApJ, 363, 603

Dai, Z. G., Peng, Q. H., \& Lu, T. 1995, ApJ, 440, 815

Danielewitz, P. 1984, Phys. Lett. B, 146, 168

Duncan, R. C., \& Thompson, C. 1992, ApJ, 392, L9

Flowers, E., \& Itoh, N. 1976, ApJ, 206, 218 1979, ApJ, 230, 847 1981, ApJ, 250, 750

Glendenning, N. K. 1992, Phys. Rev. D, 46, 1274 2000, Compact Stars, Nuclear Physics, Particle Physics and General

Relativity (New York: Springer)

Glendenning, N. K., Pei, S., \& Weber, F. 1997, Phys. Rev. Lett., 79, 1603

Grassi, F. 1998, ApJ, 492, 263

Haensel, P., Zdunik, J. L., \& Schaeffer, R. 1986, A\&A, 160, 121

Hakim, R., \& Mornas, L. 1993, Phys. Rev. C, 47, 2846

Harko, T., \& Cheng, K. S. 2000, Phys. Lett. A, 266, 249 2002, A\&A, 385, 947

Heiselberg, H. 1995, in Quark Matter Droplet Formation in Neutron Stars, International Symposium on Strangeness and Quark Matter, ed. S. Kumar \& J. Madsen (Singapore: World Scientific), 338-342

Heiselberg, H., Pethick, C. J., \& Staubo, E. F. 1993, Phys. Rev. Lett., 70, 1355

Horvath, J. E. 1994, Phys. Rev. D, 49, 5590

Horvath, J. E., \& Benvenuto, O. G. 1988, Phys. Lett. B, 213, 516

Horvath, J. E., Benvenuto, O. G., \& Vucetich, H. 1992, Phys. Rev. D, 45, 3865

Itoh, N. 1970, Prog. Theor. Phys., 44, 291

Kawasaki, K. 1975, J. Stat. Phys., 12, 365

Landau, L. D., \& Lifshitz, E. M. 1980, Statistical Physics (Oxford: Pergamon)

Langer, J. S., \& Turski, L. A. 1973, Phys. Rev. A, 8, 3230

Lattimer, J. M., \& Ravenhall, D. G. 1978, ApJ, 223, 314

Lorentz, C. P., Ravenhall, D. G., \& Pethick, C. J. 1993, Phys. Rev. Lett., 70, 379

Lugones, G., \& Benvenuto, O. G. 1995, Phys. Rev. D, 52, 1276
Lugones, G., Benvenuto, O. G., \& Vucetich, H. 1994, Phys. Rev. D, 50, 6100 Lyubarsky, Y., Eichler, D., \& Thompson, C. 2002, ApJ, 580, L69

Ma, F., \& Xie, B. 1996, ApJ, 462, L63

Madsen, J. 1993, Phys. Rev. Lett., 70, 391

Madsen, J., \& Olesen, M. L. 1991, Phys. Rev. D, 43, 1069

Manchester, R. N., \& Taylor, J. H. 1977, Pulsars (San Francisco: Freeman) Mardor, I., \& Svetitsky, B. 1991, Phys. Rev. D, 44, 878

Ng, C. Y., Cheng, K. S., \& Chu, M. C. 2003, Astropart. Phys., 19, 171

Norsen, T. 2002, Phys. Rev. C, 65, 045805

Olesen, M. L., \& Madsen, J. 1994, Phys. Rev. D, 49, 2698

Olinto, A. V. 1987, Phys. Lett. B, 192, 71

Paczynski, B. 1992, Acta Astron., 42, 145

Pandharipande, V. R. 1971, Nucl. Phys. A, 174, 641

Pandharipande, V. R., Pines, D., \& Smith, R. A. 1976, ApJ, 208, 550

Pandharipande, V. R., \& Smith, R. A. 1975, Nucl. Phys. A, 237, 507

Rajagopal, K., \& Wilczek, F. 2001, Phys. Rev. Lett., 86, 3492

Ruggeri, F., \& Friedman, W. A. 1996, Phys. Rev. D, 53, 6543

Sawyer, R. S. 1989, Phys. Lett. B, 233, 412

Shapiro, S. L., \& Teukolsky, S. A. 1983, Black Holes, White Dwarfs and Neutron Stars (New York: Wiley)

Shukla, P., \& Mohanty, A. K. 2001, Phys. Rev. C, 64, 054910

Shukla, P., Mohanty, A. K., \& Gupta, S. K. 2001, Phys. Rev. D, 63, 014012

Shukla, P., Mohanty, A. K., Gupta, S. K., \& Gleiser, M. 2000, Phys. Rev. C, 62,054904

Thompson, C., \& Duncan, R. C. 1995, MNRAS, 275, 255 1996, ApJ, 473, 322

Tsuruta, S. 1998, Phys. Rep., 292, 1

Usov, V. V. 2001, Phys. Rev. Lett., 787, 21101

Venugopalan, R., \& Vischer, A. P. 1994, Phys. Rev. E, 49, 5849

Walecka, J. D. 1975, Phys. Lett. B, 59, 109

Wiringa, R. B., Fiks, V., \& Fabrocini, A. 1988, Phys. Rev. C, 38, 1010

Witten, E. 1984, Phys. Rev. D, 30, 272 